\title{
Chinese Handwriting Signature Identification techniques
}

\author{
Yongjian Zhao \\ Information Engineering Institute, Shandong University (Weihai), China \\ jian123cn@sdu.edu.cn
}

Keywords: Signature, Feature, Formula, Performance, Property.

\begin{abstract}
Chinese handwriting signature is a useful biological feature for identity verification. This paper presents a hybrid signature identification method. Applying wavelet transform to each stroke of signature, reliable signature characteristic is extracted. Subsequently, a desirable classifier is introduced to distinguish various pseudo signatures from the real one. Then an identification method is developed correspondingly and its performance is demonstrated by computer simulations.
\end{abstract}

\section{Introduction}

Chinese handwriting signature is combined actions of psychology, physiology and optics [1]. By verifying the writer's identity on the basis of handwriting, Chinese signature identification can efficiently distinguish various pseudo signatures from the real one [2]. As an efficient approach of biometric personal identification, Chinese signature identification can be helpful for many kinds of information security systems, such as credit card transaction in electronic commerce and the authority of electronic documents. In the last decades, Chinese handwriting signature identification has become an active research topic in the domain of computer vision and pattern recognition $[3,4]$.

At present, a few signature identification methods have been presented on the basis of Fourier transform and neural networks [1-3]. But these methods have some shortcomings, such as the low identification rate, the difficulty to perform and the tendency to lose geometric qualities of the signature [5,6].

Due to perfect property about adaptive feature and mathematical microscope feature, wavelet transform has become a focus issue in the domain of signal processing. This paper presents a hybrid method to extract signature characteristic through wavelet transform. Characteristic extraction is a kernel step of Chinese signature identification, which can directly determine system performance. Chinese handwriting signature is composed of a few strokes and each stroke can be considered a curve. Spline function can efficiently deal with curve and surface in the domain of computer graphics. At first, a group of 4th order spline wavelet is presented on the basis of spline function. Subsequently, wavelet transform is applied to each stroke of signature in accordance with wavelet formula of multi-resolution analysis. The extracted signature characteristic has better properties such as rotating-invariant, translating-invariant and dilating-invariant. At last, a proper classifier is introduced to identify the signature whether it is authentic or forged through distance match. Computer simulation results show that the proposed method has better stability and reliability.

\section{Spline Wavelet Bases}

The basic principle of wavelet transform is to seek for a series of orthogonal wavelet bases, on which original signal can be decomposed and synthesized. Spline function has many desirable properties, such as recursion, local positive supported, multi-scale and the smallest compact supported [2,3].

The $m$ th order spline $\mathrm{N}_{\mathrm{m}}$ satisfies the following properties[1,6]:

(1) $N_{m}(x)=\frac{1}{(m-1) !} \Delta^{m} x_{+}{ }^{m-1}=\frac{1}{(m-1) !} \sum_{k=0}^{m}(-1)^{k}\left(\begin{array}{l}m \\ k\end{array}\right)(x-k)_{+}{ }^{m-1}$

where $\Delta f(x)=f(x)-f(x-1), x_{+}=x(x \geq 0), x_{+}=0(\mathrm{x}<0)$. 
(2) $N_{m}(x)=\frac{x}{m-1} N_{m-1}(x)-\frac{m-x}{m-1} N_{m-1}(x-1)$.

(3) The Fourier transform of $N_{1}(x)$ is $\hat{N}_{1}(\omega)=\frac{1-e^{i \omega}}{i \omega}=e^{-\frac{i \omega}{2}}\left[\frac{\sin (\omega / 2)}{\omega / 2}\right]$.

(4) The Fourier transform of $N_{m}(x)$ is $\hat{N}_{m}(\omega)=\left(\frac{1-e^{i \omega}}{i \omega}\right)^{m}=e^{-\frac{i m \omega}{2}}\left[\frac{\sin (\omega / 2)}{\omega / 2}\right]^{m}$.

Here we introduce a function as $\phi_{1}(x)=N_{m}(x+m / 2)$, whose Fourier transform is $\hat{\phi}_{1}(\omega)=\left[\frac{\sin (\omega / 2)}{\omega / 2}\right]^{m}$.

Suppose that $e_{n}(\omega)=\sum_{k=-\infty}^{+\infty} \frac{1}{(\omega+2 k \pi)^{n+2}}$, one can deduce that

$e_{n}{ }^{1}(\omega)=-(n+2) \sum_{k=-\infty}^{+\infty} \frac{1}{(\omega+2 k \pi)^{n+2+1}}=-(n+2) e_{n+1}(\omega)$ and $e_{n+1}(\omega)=-\frac{1}{n+2} e_{n}^{1}(\omega)$.

Since $e_{0}(\omega)=\sum_{k=-\infty}^{+\infty} \frac{1}{(\omega+2 k \pi)^{2}}=\frac{1}{4 \sin ^{2}(\omega / 2)}$, one can deduce that

$e_{n+1}(\omega)=-\frac{1}{n+2} e_{n}^{1}(\omega)$ and $\sum_{k=-\infty}^{+\infty}|\hat{\phi}(\omega+2 k \pi)|^{2}=2^{2 m} \sin ^{2 m}\left(\frac{m}{2}\right) e_{2(m-1)}(\omega)$.

So $\hat{\phi}(\omega)=\left[\frac{\sin (\omega / 2)}{\omega / 2}\right]^{m} \cdot \frac{1}{\sin ^{m}(\omega / 2)} \cdot \frac{1}{\sqrt{\sum_{k=-\infty}^{+\infty} \frac{1}{(\omega+2 k \pi)^{2 m}}}}=\frac{1}{\omega^{m}} \cdot \frac{1}{\sqrt{e_{2(m-1)}(\omega)}}$.

Because $\hat{\phi}(2 \omega)=H(\omega) \hat{\phi}(\omega)$, one can deduce that $H(\omega)=2^{-m} \sqrt{\frac{e_{2(m-1)}(\omega)}{e_{2(m-1)}(2 \omega)}}$.

The discrete inverse Fourier transform of $\mathrm{H}(\omega)$ is impulse response $\left\{\mathrm{h}_{1}\right\} \mathrm{l}=0 \cdots 511$ from scaling function $\psi(\mathrm{t})$, whose value can be calculated. Due to space constraint, the values of $h_{1}$ and $g_{1}$ (l=0..511) are omitted here where $\mathrm{g}_{\mathrm{k}}=(-1)^{\mathrm{k}-1} \mathrm{~h}_{512-\mathrm{k}} \mathrm{k}=1 \ldots .511$ and $\mathrm{g}_{0}=(-1) \mathrm{h}_{1}$.

After preprocessing, each stroke of specific signature can be expressed by 512 discrete complex number[1,2,6]. Supposed that the signature have $M$ strokes, the $m$ th stroke can be described as

$\left\{\mathrm{f}_{\mathrm{m}}(\mathrm{k})=\mathrm{x}_{\mathrm{m}}(\mathrm{k})+\mathrm{i} \mathrm{y}_{\mathrm{m}}(\mathrm{k}) \quad \mathrm{k}=0,1, \ldots, \mathrm{N}-1 \mathrm{~N}=512 \mathrm{~m}=0,1, \ldots, \mathrm{M}-1\right\}$.

Multi-resolution of above stroke can be deduced as

$$
\begin{aligned}
A_{j}^{d} f_{m}(n) & =\sum_{k=0}^{2^{J+1} N-1} h(l) A_{j+1}^{d} f_{m}(k)=\sum_{k=0}^{2^{j+1} N-1} h(l) A_{j+1}^{d} x_{m}(k)+i \sum_{k=0}^{2^{j+1} N-1} h(l) A_{j+1}^{d} y_{m}(k) \text { and } \\
D_{j}^{d} f_{m}(n) & =\sum_{k=0}^{2^{j+1} N-1} g(l) A_{j+1}^{d} f_{m}(k)=\sum_{k=0}^{2^{j+1} N-1} g(l) A_{j+1}^{d} x_{m}(k)+i \sum_{k=0}^{2^{j+1} N-1} g(l) A_{j+1}^{d} y_{m}(k),
\end{aligned}
$$

where $l=[k-2(2 n+1)] \bmod \left(2^{j+1} N\right) n=0, \ldots, 2^{j} N-1 j=-1,-2 \ldots-J \quad J>0 \quad A_{0}{ }^{d} f_{m}(K)=f_{m}(K)=x_{m}(K)+i y_{m}(k)$ $\mathrm{k}=0 \ldots \mathrm{N}-1$.

When decomposed to $j$ th layer, the sample number of $A_{j}{ }^{d} f_{m}(k)$ and $D_{j}{ }^{d} f_{m}(k)$ is $2^{j} N$ respectively where $\mathrm{j}<0$ and $\mathrm{k}=0, \ldots, 2^{\mathrm{j}} \mathrm{N}$. Correspondingly, while decomposed to Jth layer, wavelet representation of this stroke can be further described as

$\left\{\left\{\mathrm{A}_{-\mathrm{J}}{ }^{\mathrm{d}} \mathrm{f}_{\mathrm{m}}(\mathrm{k})\right.\right.$ where $\left.\mathrm{k}=0 \ldots 2^{-\mathrm{J}} \mathrm{N}\right\}$ and $\left\{\mathrm{D}_{\mathrm{j}}{ }^{\mathrm{d}} \mathrm{f}_{\mathrm{m}}(\mathrm{k})\right.$ where $\left.\left.\mathrm{j}=-\mathrm{J},-\mathrm{J}+1, \ldots,-1 \mathrm{k}=0 \ldots 2^{\mathrm{j}} \mathrm{N}\right\}\right\}$.

Here we introduce a series of wavelet formulas to synthesis characteristic of a signature on the basis of discrete complex wavelet transform [1,2].

For $A_{-J}{ }^{d} f_{m}(k)$, we set 


$$
A_{-J}^{d} \bar{x}_{m}=\frac{1}{2^{-J} N} \sum_{K=0}^{2^{-J} N-1} A_{-J}{ }^{d} x_{m}(k) \text { and } A_{-J}^{d} \bar{y}_{m}=\frac{1}{2^{-J} N} \sum_{K=0}^{2^{-J}} A_{-J}{ }^{d} y_{m}(k)
$$

where $\mathrm{j}=-\mathrm{J},-\mathrm{J}+1, \ldots,-1 \mathrm{~m}=0, \ldots, \mathrm{M}-1$.

For $\mathrm{D}_{\mathrm{j}} \mathrm{d}_{\mathrm{m}}(\mathrm{k})$, we set

$$
D_{j}^{d} \bar{x}_{m}=\frac{1}{2^{-J} N} \sum_{K=0}^{2^{-J}} D_{j}^{d} x_{m}(k) \text { and } D_{j}^{d} \bar{y}_{m}=\frac{1}{2^{-J} N} \sum_{K=0}^{2^{-J}} D_{j}^{d} y_{m}(k)
$$

where $\mathrm{j}=-\mathrm{J},-\mathrm{J}+1, \ldots,-1 \mathrm{~m}=0,1, \ldots, \mathrm{M}-1$.

For $F A_{-J}^{d} f_{m}$, we set

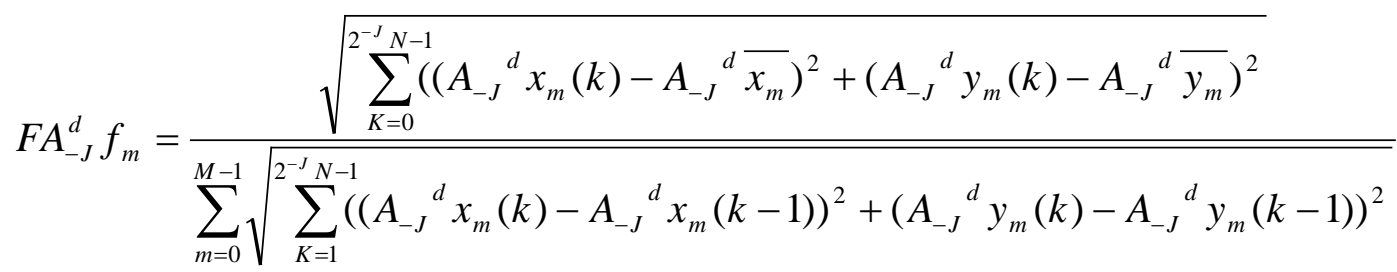

where $\mathrm{j}=-\mathrm{J},-\mathrm{J}+1, \ldots,-1 \mathrm{~m}=0,1, \ldots, \mathrm{M}-1$.

For $F D_{j}^{d} f_{m}$, we set

$$
F D_{j}^{d} f_{m}=\frac{\sqrt{\sum_{K=0}^{2^{-J}}\left(\left(D_{j}{ }^{d} x_{m}(k)-D_{j}{ }^{d} \overline{x_{m}}\right)^{2}+\left(D_{j}{ }^{d} y_{m}(k)-D_{j}{ }^{d} \overline{y_{m}}\right)^{2}\right.}}{\sum_{m=0}^{M-1} \sqrt{\sum_{K=1}^{2^{-J} N-1}\left(\left(D_{j}{ }^{d} x_{m}(k)-D_{j}{ }^{d} x_{m}(k-1)\right)^{2}+\left(D_{j}{ }^{d} y_{m}(k)-D_{j}{ }^{d} y_{m}(k-1)\right)^{2}\right.}}
$$

where $\mathrm{j}=-\mathrm{J},-\mathrm{J}+1, \ldots,-1 \mathrm{~m}=0,1, \ldots, \mathrm{M}-1$.

Above synthesis formulas have desirable properties, such as rotation invariance, transformation invariance and scale invariance. The characteristics of the mth stroke are $\left\{F A_{-J}{ }^{d} f_{m}, F D_{j}{ }^{d} f_{m}\right.$ $\mathrm{j}=-\mathrm{J},-\mathrm{J}+1, \ldots,-1\}$. If we seek $\left\{F A_{-5}{ }^{d} f_{m}, F D_{j}{ }^{d} f_{m} \mathrm{j}=-5,-4,-3\right\}$ as characteristic of the mth stroke, the whole signature characteristic can be described as $\left\{F A_{-5}{ }^{d} f_{m}, F D_{j}{ }^{d} f_{m} \mathrm{j}=-5,-4,-3 \mathrm{~m}=0,1, \ldots, \mathrm{M}-1\right\}$.

\section{Matching Identification}

After a series of stable feature values of the signature is extracted, one can utilize distance match to identify whether a specific signature is authentic or forged. The classical distance match method is Euclidean distance, which can be expressed as the following equation:

$$
D(x, y)=\sqrt{\left(x_{1}-y_{1}\right)^{2}+\cdots\left(x_{n}-y_{n}\right)^{2}}=\sqrt{(x-y)^{\prime}(x-y)},
$$

where $\mathrm{x}$ and y are two points in $R^{n}$.

Euclidean distance sometimes is not appropriate in multidimensional analysis or statistical calculations. Suppose that there are two normal populations $\left(\left(\pi_{1} \sim N\left(\mu_{1}, \delta_{1}^{2}\right), \pi_{2} \sim N\left(\mu_{2}, \delta_{2}^{2}\right)\right)\right.$ and a sample is set in A, which normal population the sample is closer to ?

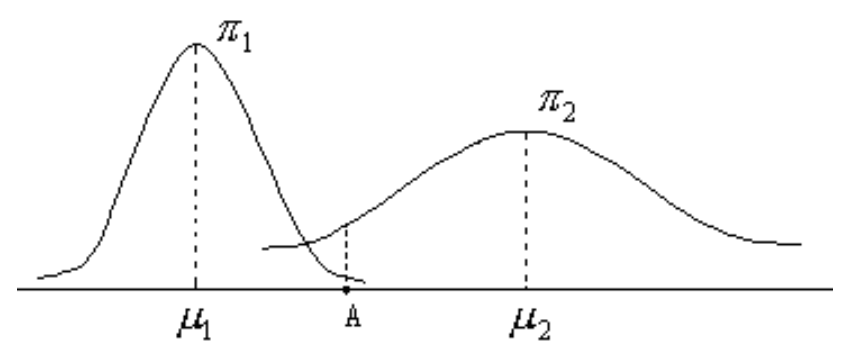


Through Euclidean distance, point $\mathrm{A}$ is a little closer from the center $\mu_{1}$ of $\pi_{1}$ than it is from the center $\mu_{2}$ of $\pi_{2}$. However, from the perspective of probability theory, point A is at about $4 \sigma_{1}$ the right of $\mu_{1}$ and about $25 \sigma_{2}$ the left of $\mu_{2}$. Through standard deviation to measure, point $\mathrm{A}$ is a little closer from $\mu_{2}$ than from $\mu_{1}$. Obviously, the latter measurement is more reasonable.

The Mahalanobis distance between point $\mathrm{x}$ and the class $\pi$ is defined as

$$
D(x, \pi)=\left[(x-\mu)^{\prime} \sum^{-1}(x-\mu)\right]^{\frac{1}{2}}
$$

where $\mu$ is the mean values of $\pi$ and $\sum$ is the covariance matrix. Suppose that the mean vectors of class $\pi_{1}$ and class $\pi_{2}$ are $\mu_{1}$ and $\mu_{2}$ respectively, the covariance matrix are $\sum_{1}$ and $\sum_{2}$ respectively. Now given an individual $\mathrm{x}$, we should determine which class $\mathrm{x}$ comes from. After calculating Mahalanobis distance from $\mathrm{x}$ to class $\pi_{1}$ and $\pi_{2}$, we can compare the two distances $\mathrm{D}(\mathrm{x}$, $\left.\pi_{1}\right)$ and $\mathrm{D}\left(\mathrm{x}, \pi_{2}\right)$. If $\mathrm{D}\left(\mathrm{x}, \pi_{1}\right)<\mathrm{D}\left(\mathrm{x}, \pi_{2}\right)$, one can deduce that $\mathrm{x}$ belongs to class $\pi_{1}$, otherwise $\mathrm{x}$ belongs to class $\pi_{2}$.

Table 1 Typical identification results

\begin{tabular}{|c|c|c|}
\hline Sample library & Mean distinction rate(\%) & Optimum distinction rate(\%) \\
\hline Method in this paper & 91.7 & 92.8 \\
\hline Random choice & 83.6 & 88.7 \\
\hline
\end{tabular}

From Table 1, one can find that the method proposed in this paper is more effective and stable than the traditional method. Indeed, after stable feature extraction, the samples' distribution is more reasonable than before and the samples are more likely to assemble to the mean value. In other words, the samples tend to be act as normal distribution.

\section{Conclusions}

As combined actions of psychology, physiology and optics, Chinese handwriting signature has become an important approach for biometric personal identification. Based on wavelet transform, a hybrid signature identification method is proposed in this paper. As this method constructs the 4th B-spline wavelet and applies wavelet transform to each stroke of a signature, the extracted signature characteristic is more effective and reliable, thus enhancing the effects of identification. The appropriate classifier is developed and further improves the identification performance. The theoretical framework developed here should provide a strong foundation for future research and application.

\section{References}

[1] Zhaoqi Bian, Pattern Recognition, the Tsinghua University Press(1994)

[2] Yongjian Zhao, Chinese writing identification based on wavelet transform, Computer Project, 8:135-147(2005)

[3] B D Ripley:Pattern Recognition and Neural Networks, Cambrige University Press, Cambrige (2006)

[4] Bovik A C,Gopal N, Emmoth T, Localized measurement of emergent image frequencies by Gabor wavelets, IEEE Trans. Inf. Theory, 56 (2):691-712(2004)

[5] ZhenYu He,Qinghu Chen, A neural network expert system for Chinese handwriting-based writer identification, Machine Learning and Cybernetics(2002)

[6] He,Z.Y., Tang,Y.Y., Chinese handwriting-based writer identification by texture analysis , Machine Learning and Cybernetics(2004) 